\title{
IMPACTO DE "EL NIÑO 1997-98”" SOBRE EL ECOSISTEMA MARINO SOMERO DE LA COSTA CENTRAL DEL PERÚ
}

\author{
IMPACT OF "EL NIÑO 1997-98" ON SHALLOW MARINE ECOSYSTEM \\ OF THE PERUVIAN CENTRAL COAST
}

\author{
Juan Tarazona, Aldo Indacochea, Sonia Valle, César Córdova, Noemí Ochoa, \\ Wilbert Serrano y Tania Peña*
}

\section{RESUMEN}

Se analizó las caracteristicas de intensidad y precocidad de las respuestas a "El Niño 1997-98" manifestada por el fitoplancton superficial, macroalgas de la orilla rocosa y macrobentos de fondo blando

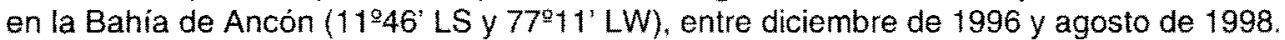

Este evento se caracterizó por un incremento significativo de la temperatura desde abril $\left(17,4^{\circ} \mathrm{C}\right)$ y del oxígeno $\left(2,46 \mathrm{ml} \times \mathrm{l}^{-1}\right)$ a partir de mayo de 1997, en los fondos del área de estudio. En el fitoplancton superficial se registró la presencia de especies indicadoras de aguas oceánicas cálidas desde enero y un inusual aumento en la densidad de dinoflagelados y microflagelados a inicios de abril de 1997.

La densidad de la población de la macroalga Chondracanthus chamissoi alcanzó 2684 ind. $\times 32 \mathrm{~m}^{-1}$ de línea de orilla en octubre de 1997, y la proporción de gametofitos se incrementó durante 1998. La densidad y el número de especies del macrobentos de fondo blando alcanzaron los valores máximos de 90 ind $\times 0,04 \mathrm{~m}^{2}$ y 20 especies en junio y noviembre de 1997, respectivamente; estas variaciones correlacionaron bien con los cambios en la intensidad de "El Niño". La biomasa llegó a su máximo valor de $0,55 \mathrm{~g}$ pssc $\times 0,04 \mathrm{~m}^{-2}$ recién en enero de 1998 .

Finalmente, se demuestra que el impacto biológico de "El Niño 1997-98" fue menor al del evento de 1982-83, no obstante la similitud en la intensidad de ambos eventos; y que las aguas someras presentaron indicadores tempranos y confiables de "El Niño", tanto en el fitoplancton como en el bentos. Además, la presencia de un período post-Niño demuestra que el impacto de "El Niño" sobre las poblaciones y comunidades puede ser complejo.

Palabras clave: Ecosistema marino, macrobentos, fitoplancton, Bahia de Ancón, El Niño 1997-98.

\section{ABSTRACT}

The characteristics of intensity, and untimely of "El Niño 1997-98" answer, manifested by superficial phytoplankton, rocky shore macroalgae, and soft bottom macrobenthos were analyzed from December

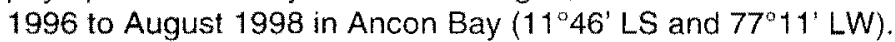

A significative increase of both, temperature since April $\left(17,4^{\circ} \mathrm{C}\right)$, and oxygen $\left(2,46 \mathrm{ml} \times \mathrm{l}^{-1}\right)$ from May 1997 in the bottom of the study area, characterized this event. In superficial phytoplankton, the presence of indicator species of warm oceanic water, and an unusual increase of both dinoflagellate and microflagellate density at the beginning of April 1997 were recorded since January.

The density of Chondracanthus chamissoi population reached 2684 ind. $\times 32 \mathrm{~m}^{\prime \prime}$ of shoreline in October 1997, and the proportion of gametophytes increased during 1998. The density and species number of soft bottom macrobenthos reached maximum values of 90 ind. $x 0,04 \mathrm{~m}^{-2}$, and 20 species in June, and November 1997 respectively; these variations have a good correlation with intensity changes of "El Niño". The biomass reached its maximum value $0,55 \mathrm{~g} \mathrm{pssc} \times 0,04 \mathrm{~m}^{2}$ in January 1998 .

Finally, it is showed that "El Niño 1997-98" biological impact, was lesser than that of 1982-83 however the similarity of intensity in both events; and that shallow water showed early and reliable indicators of "EI

* Grupo DePSEA, Fac. de Ciencias Biológicas - UNMSM. Apartado 1898, Lima-100, Perú.

Red sobre Impacto Biológico de los Eventos EI Niño (RIBEN) - CONCYTEC. 
Niño", in both, the phytoplankton as in the benthos. Moreover, the presence of a post-Niño period showed that "El Niño" impact on population and communities might be complex.

Key words: Marine ecosystem, macrobenthos, phytoplankton, Ancon Bay, El Niño 1997-98.

\section{INTRODUCCIÓN}

El ecosistema de surgencias costero peruano ha sido conocido como el más productivo del mundo (Rither, 1969; Barber y Kogelschatz, 1990). Sin embargo, también se caracteriza por fluctuaciones muy grandes en la abundancia y distribución de los recursos (Schweigger, 1964; Majluf y Trillmich, 1981; Santander y Tsukuyama, 1984; Espino et al., 1985; Rojas de Mendiola, 1985; Carrasco y Lozano, 1989; Tarazona et al., 1996). A escala interanual, la mayor parte de esta variabilidad es producida por los eventos "El Niño" (Enfield y Cid, 1991). En consecuencia, para el desarrollo de todas las actividades económicas relacionadas directa o indirectamente con el aprovechamiento de los recursos marinos en nuestro país, se requiere una buena capacidad de predicción temprana de los impactos negativos o positivos de "El Niño" (EN); así como el desarrollo de una mayor capacidad de manejo del ecosistema con el fin de prolongar los efectos positivos y aminorar los negativos.

En las últimas décadas, cada tres a cuatro años se ha presentado un evento EN de diversa intensidad. El evento EN 1982-83 había sido considerado como el más intenso del presente siglo con anomalías térmicas de cerca de $11^{\circ} \mathrm{C}$ por encima del promedio patrón, se consideraba que eventos extremadamente fuertes se presentaban sólo después de varias décadas, rememorando el evento de 1925. Sin embargo, recientemente, en poco más de una década, se presentó un nuevo evento muy fuerte $\mathrm{EN}$ 1997-98, que en algunos aspectos incluso superó en intensidad al evento 198283 (Jaimes, en este volumen). Este fue particularmente diferente a los otros eventos porque sus manifestaciones en la costa sudamericana se iniciaron en otoño.
Por lo cual, en el presente trabajo se trata de establecer las particularidades del impacto biológico de EN $1997-98$ sobre el ecosistema marino somero de la costa central del Perú, evaluando la variación temporal del macrobentos de fondo blando, fitoplancton y macroalgas de orilla rocosa asociada a la variabilidad oceanográfica inducida por $\mathrm{EN}$; discriminando también las interacciones biológicas que cumplieron un papel destacado en la estructuración de las comunidades y poblaciones.

\section{MATERIAL Y MÉTODOS}

El estudio fue desarrollado en la parte sur de la Bahía de Ancón, situada a unos $40 \mathrm{~km}$ al norte de Lima, donde se estableció cuatro estaciones fijas: la estación $\mathrm{B}$ de $15 \mathrm{~m}$ de profundidad, la estación $\mathrm{C}$ de $34 \mathrm{~m}$ de profundidad, y las estaciones $D$ y $S$ que abarcaron cada una $32 \mathrm{~m}$ de línea costera en la orilla rocosa (Fig. 1).

Los datos oceanográficos, por lo general, fueron tomados mensual y quincenalmente (en la estación C), desde una embarcación de pesca artesanal. Se tomó temperatura y muestras de agua para la determinación de oxígeno disuelto en tres niveles de profundidad (superficie, media agua y fondo), utilizando una botella Niskin provista de un termómetro de inversión. El análisis de la concentración de oxigeno disuelto se realizó utilizando el método de Winkler modificado.

Los muestreos quincenales de fitoplancton superficial (en la estación B) fueron realizados desde una embarcación de pesca artesanal. En cada fecha se obtuvo una muestra de $200 \mathrm{ml}$, utilizando una botella Niskin. Las muestras ya fijadas en formalina fueron analizadas según el método de Uthermöhl modificado, 
Figura 1. Mapa de la Bahía de Ancón. Ubicación de las estaciones de muestreo: $\mathrm{B}$ y $\mathrm{C}$ (15 y $34 \mathrm{~m}$ de profundidad, respectivamente), D y S (32 m de línea de orilla).

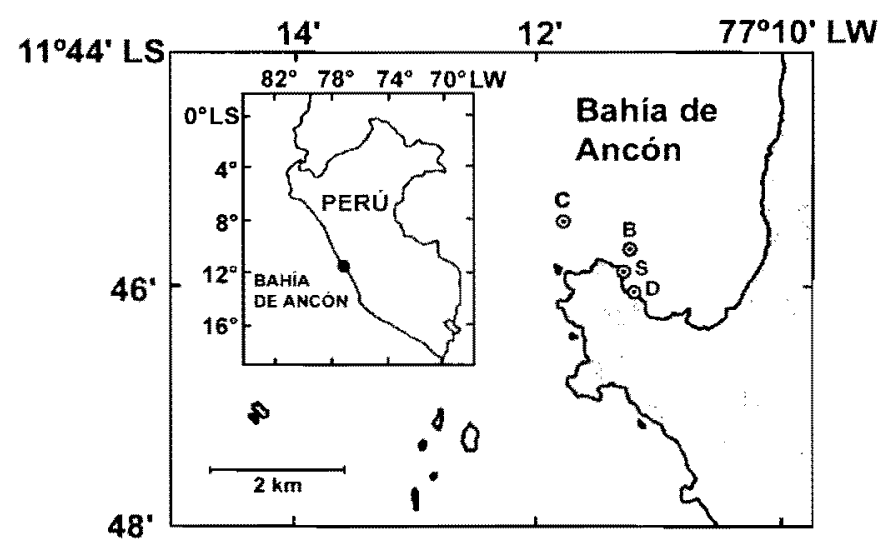

de la estructura comunitaria del macrobentos de fondo blando, a lo largo del período de estudio, fueron realizados utilizando los indices de similaridad de las densidades. El método de grupos de pares no balanceados usando promedios aritméticos (UPGMA) fue empleado en los análisis de clasificación (Sneath y Sokal, 1973); y el escaleo no métrico multidimensional (NMDS) como técnica de ordenación (Fasham, 1977; Prentice, 1977).

El índice multivariado del impacto biológico de "El Niño" (IMIBEN) se obtuvo en base a la integración de las primeras dos dimensiones del escaleo no métrico multidimensional. Los valores positivos del índice corresponden al estado "El Niño" de la comunidad y los valores negativos al estado "La Niña".

La representación gráfica de los datos de macrobentos, tanto de orilla rocosa, como de fondo blando son presentados como promedios y se incluye los límites de error estándar $( \pm$ S.E.). El periodo de estudio fue dividido en 4 etapas de acuerdo a la variabilidad térmica de las aguas costeras de la costa central: la etapa antes de EN (diciembre de 1996 - abril de 1997); la etapa del primer pico de calentamiento de EN (mayo - octubre de 1997); la etapa del segundo pico de calentamiento de EN (noviembre de 1997 - junio de 1998); y la etapa post-Niño (julio agosto de 1998). Para analizar el efecto de cada 
una de las etapas del calentamiento de $\mathrm{EN}$ 1997-98 se desarrolló el análisis de varianza para las medias de las variables biológicas estudiadas, con el $95 \%$ de confiabilidad; y posteriormente, se discriminó los grupos homogéneos mediante el análisis de múltiples rangos, al $95 \%$ de confiabilidad, con la prueba de Scheffe.

\section{RESULTADOS}

\section{Condiciones oceanográficas}

El calentamiento de las aguas costeras en la Bahía de Ancón mostró un comportamiento particular durante "El Niño 1997-98". En febrero, abril y junio de 1997 los incrementos de temperatura superficial alcanzaron entre 21,1 y $22,0^{\circ} \mathrm{C}$; y a partir de junio, el incremento de temperatura se generalizó a toda la columna de agua. La temperatura de fondo mostró un acelerado incremento desde abril, mes en que se registró $17,4^{\circ} \mathrm{C}$ (Fig. 2). A partir de agosto de 1997, los valores de temperatura empezaron a disminuir, pero en octubre reinician su ascenso. Entre diciembre de 1997 y febrero de 1998, aparecen los valores máximos del segundo pico de calentamiento de "El Niño", alcanzando entre 24,4 y $25,5^{\circ} \mathrm{C}$ en superficie, posteriormente la temperatura fue declinando rápidamente hasta agosto de 1998 (Fig. 2).

Las concentraciones de oxígeno disuelto fueron bajas en toda la columna de agua durante 1996, con valores generalmente menores a $0,5 \mathrm{ml} \times \mathrm{l}^{-1}$ en el fondo. A inicios de abril de 1997 se presentó un incremento atípico de oxígeno disuelto en las aguas superficiales (hasta $7,44 \mathrm{ml} \times \mathrm{I}^{-1}$ ); y desde inicios de mayo el incremento se generalizó a toda la columna de agua de la estación $\mathrm{C}$, registrándose en el fondo $2,46 \mathrm{ml} \times \mathrm{V}^{-1}$ (Fig. 2). La concentración empezó a disminuir a partir de agosto, pero en octubre ascendió nuevamente, llegando a concentraciones por encima de $4 \mathrm{ml} \times \mathrm{l}^{1}$, incluso en el fondo. Es importante destacar que entre abril y junio de
1998 la concentración de oxígeno disuelto en las aguas superficiales se incrementó nuevamente hasta valores de $8,04 \mathrm{ml} \times \mathrm{I}^{-1}$. Por el contrario, a media agua y en el fondo la concentración fue disminuyendo paulatinamente desde octubre de 1997. A partir de mediados de junio de 1998 las concentraciones de oxígeno disuelto en el fondo declinaron abruptamente para mantenerse en valores por debajo de $0,5 \mathrm{ml} \times \mathrm{l}^{-1}$ (Fig. 2).

\section{Fitoplancton de aguas someras}

El fitoplancton superficial de la Bahía de Ancón, durante el verano de 1997, mostró densidades bajas, con valores menores de 750000 células $\times 50 \mathrm{ml}^{-1}$. La densidad máxima se presentó a inicios de abril, a consecuencia de una inusual dominancia de microflagelados y dinoflagelados (Fig. 3), que habitualmente se manifiesta al inicio de eventos "El Niño". Sin embargo, ya desde enero de 1997 fue frecuente la presencia de especies indicadoras de aguas oceánicas cálidas, como: Emiliana huxleyii, Pyrocystis Lunula, Thalassiothrix longissima y Planktoniella sol. Durante 1998 las densidades de fitoplancton superficial fueron fluctuantes y el ciclo anual empezó a normalizarse, aunque con densidades más bajas que las normales. En general, con muy pocas excepciones las diatomeas fueron dominantes durante todo el período de estudio (Fig. 3), destacando Leptocylindrus danicus, Skeletonema costatum, Lauderia annulata, Eucampia zoodiacus y Asterionellopsis glacialis. Los pulsos de dinoflagelados, frecuentes durante EN, sólo fueron observadas hasta mayo de 1998.

\section{La población de Chondracanthus chamissoi}

La densidad total de la población del alga roja $C$. chamissoi se incrementó de 610 ind. $\times 32 \mathrm{~m}^{-1}$ de línea de orilla a 2684 durante "El Niño". En 1996 la densidad tanto 

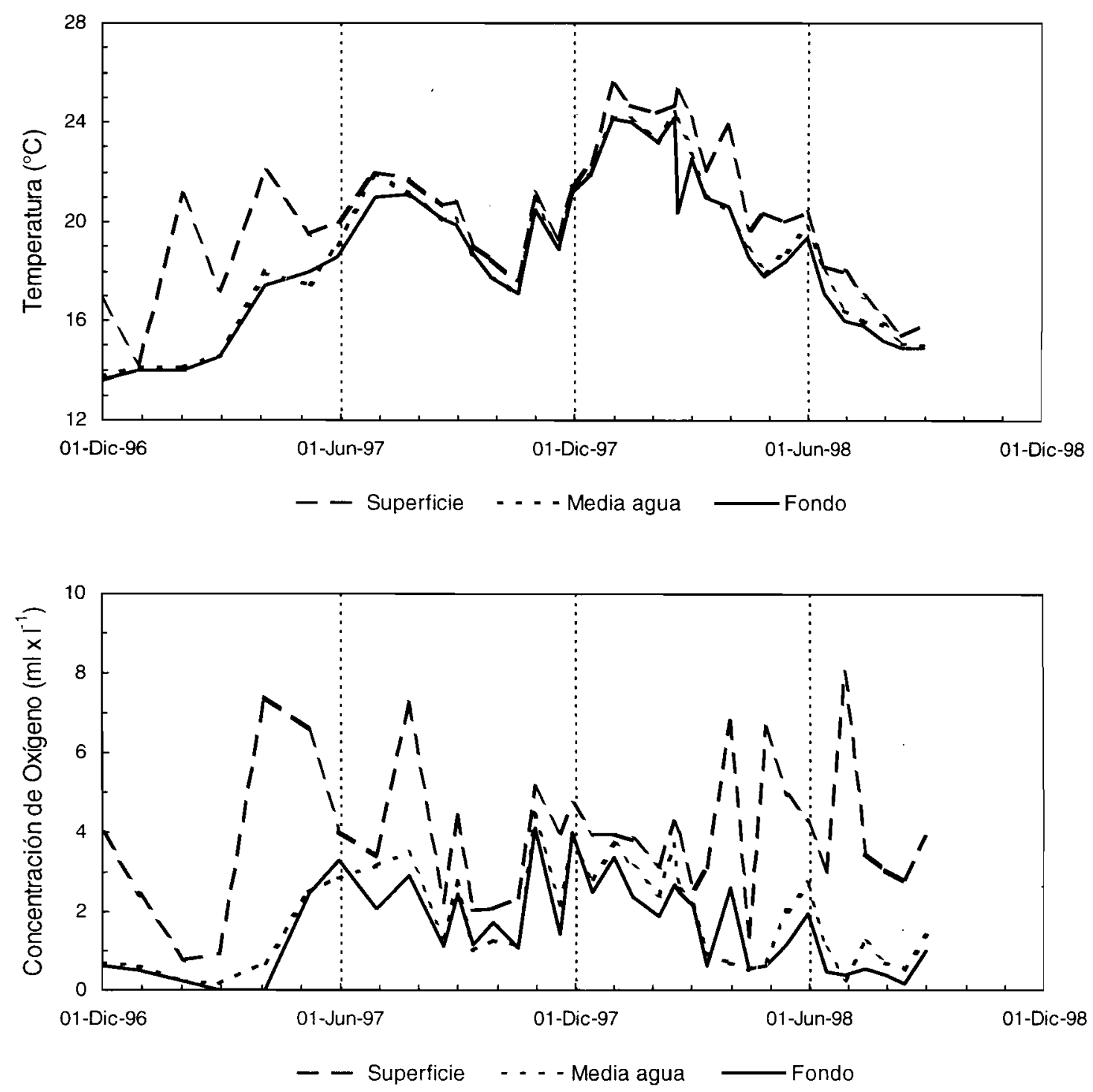

Figura 2. Variaciones de la temperatura (arriba) y concentración de oxígeno disuelto (abajo) a tres niveles de la columna de agua en la estación "C" de la Bahía de Ancón.

de plantas gametofíticas como tetrasporofíticas fue menor de 100 ind. $\times 32 \mathrm{~m}^{-1}$ de línea de orilla, con un incremento entre agosto y setiembre. A inicios de 1997, estas densidades llegaron a valores mucho mayores y mantuvo este nivel de densidades hasta octubre, cuando se produjo un incremento abrupto, que alcanzó 1815 individuos gametofíticos y 869 tetrasporofíticos en $32 \mathrm{~m}$ de línea de orilla (Fig. 4). Posteriormente, los valores de densidad empezaron a descender llegando hasta sólo 6 plantas gametofíticas y ausencia de tetrasporofíticas en enero de 1998. Esta caída de la población coincidió con la proliferación de las poblaciones de los opistobranquios Aplysia inca y A. nigra. Los valores bajos de densidad se mantuvieron durante la primera mitad de 1998, y en agosto y setiembre se manifestó un evidente incremento.

La proporción de gametofitos varió entre 
una de las etapas del calentamiento de EN 1997-98 se desarrolló el análisis de varianza para las medias de las variables biológicas estudiadas, con el $95 \%$ de confiabilidad; y posteriormente, se discriminó los grupos homogéneos mediante el análisis de múltiples rangos, al $95 \%$ de confiabilidad, con la prueba de Scheffe.

\section{RESULTADOS}

\section{Condiciones oceanográficas}

El calentamiento de las aguas costeras en la Bahía de Ancón mostró un comportamiento particular durante "El Niño 1997-98". En febrero, abril y junio de 1997 los incrementos de temperatura superficial alcanzaron entre 21,1 y $22,0^{\circ} \mathrm{C}$; y a partir de junio, el incremento de temperatura se generalizó a toda la columna de agua. La temperatura de fondo mostró un acelerado incremento desde abril, mes en que se registró $17,4^{\circ} \mathrm{C}$ (Fig. 2). A partir de agosto de 1997, los valores de temperatura empezaron a disminuir, pero en octubre reinician su ascenso. Entre diciembre de 1997 y febrero de 1998, aparecen los valores máximos del segundo pico de calentamiento de "El Niño", alcanzando entre 24,4 y $25,5^{\circ} \mathrm{C}$ en superficie, posteriormente la temperatura fue declinando rápidamente hasta agosto de 1998 (Fig. 2).

Las concentraciones de oxígeno disuelto fueron bajas en toda la columna de agua durante 1996, con valores generalmente menores a $0,5 \mathrm{ml} \times \mathrm{I}^{-1}$ en el fondo. A inicios de abril de 1997 se presentó un incremento atipico de oxigeno disuelto en las aguas superficiales (hasta $7,44 \mathrm{ml} \times \mathrm{l}^{-1}$ ); y desde inicios de mayo el incremento se generalizó a toda la columna de agua de la estación $\mathrm{C}$, registrándose en el fondo $2,46 \mathrm{ml} \times \mathrm{l}^{-1}$ (Fig. 2). La concentración empezó a disminuir a partir de agosto, pero en octubre ascendió nuevamente, llegando a concentraciones por encima de $4 \mathrm{ml} \times \mathrm{l}^{-1}$, incluso en el fondo. Es importante destacar que entre abril y junio de
1998 la concentración de oxígeno disuelto en las aguas superficiales se incrementó nuevamente hasta valores de $8,04 \mathrm{ml} \times \mathrm{I}^{-1}$. Por el contrario, a media agua y en el fondo la concentración fue disminuyendo paulatinamente desde octubre de 1997. A partir de mediados de junio de 1998 las concentraciones de oxígeno disuelto en el fondo declinaron abruptamente para mantenerse en valores por debajo de $0,5 \mathrm{ml} \times \mathrm{I}^{-1}$ (Fig. 2).

\section{Fitoplancton de aguas someras}

El fitoplancton superficial de la Bahía de Ancón, durante el verano de 1997, mostró densidades bajas, con valores menores de 750000 células $\times 50 \mathrm{ml}^{-1}$. La densidad máxima se presentó a inicios de abril, a consecuencia de una inusual dominancia de microflagelados y dinoflagelados (Fig. 3), que habitualmente se manifiesta al inicio de eventos "El Niño". Sin embargo, ya desde enero de 1997 fue frecuente la presencia de especies indicadoras de aguas oceánicas cálidas, como: Emiliana huxleyii, Pyrocystis lumula, Thalassiothrix longissima y Planktoniella sol. Durante 1998 las densidades de fitoplancton superficial fueron fluctuantes y el ciclo anual empezó a normalizarse, aunque con densidades más bajas que las normales. En general, con muy pocas excepciones las diatomeas fueron dominantes durante todo el período de estudio (Fig. 3), destacando Leptocylindrus danicus, Skeletonema costatum, Lauderia annulata, Eucampia zoodiacus y Asterionellopsis glacialis, Los pulsos de dinoflagelados, frecuentes durante $\mathrm{EN}$, sólo fueron observadas hasta mayo de 1998.

\section{La población de Chondracanthus chamissoi}

La densidad total de la población del alga roja C. chamissoi se incrementó de 610 ind $\times 32 \mathrm{~m}^{-1}$ de línea de orilla a 2684 durante "E1 Niño". En 1996 la densidad tanto 

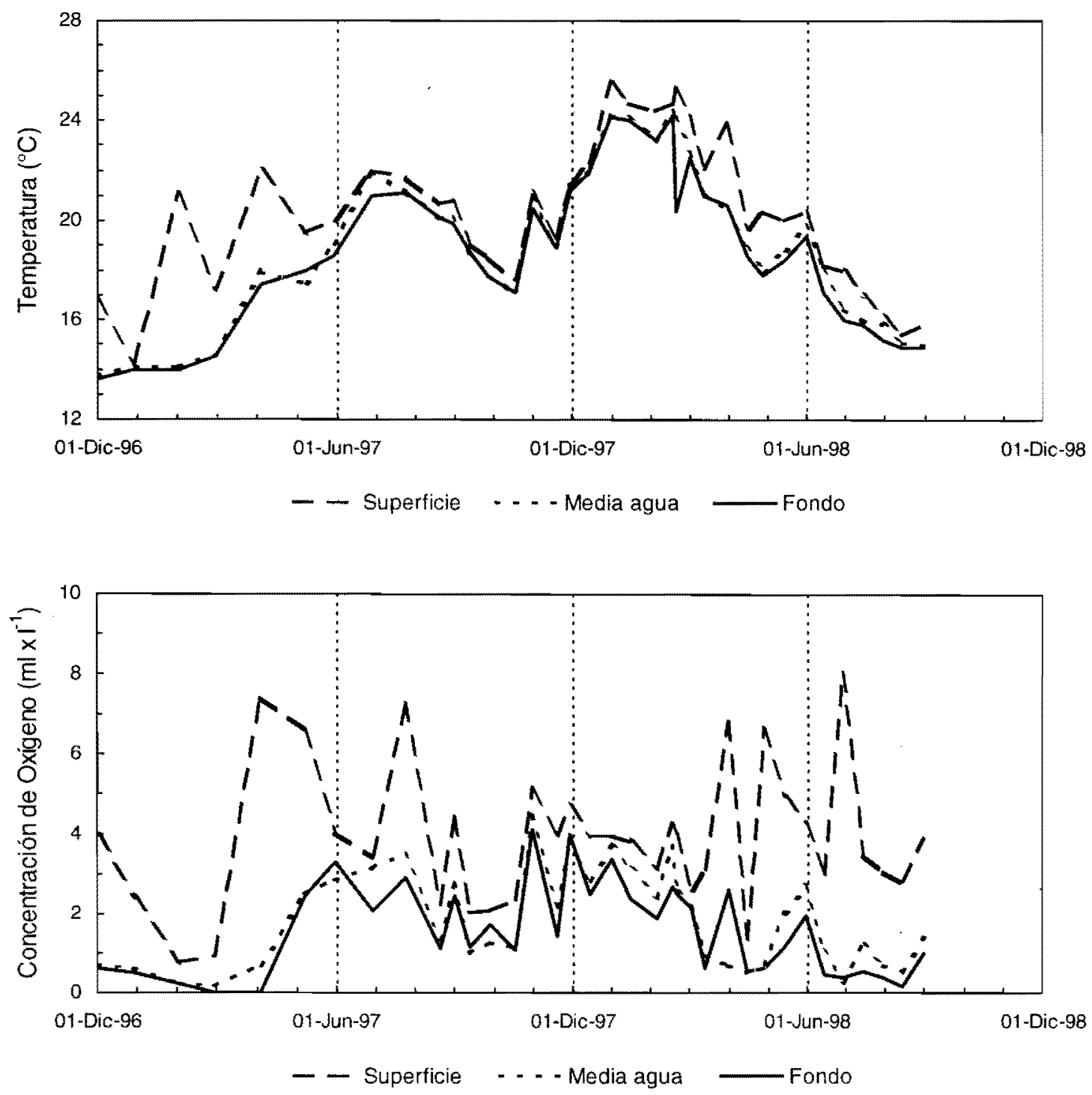

Figura 2. Variaciones de la temperatura (arriba) y concentración de oxígeno disuelto (abajo) a tres niveles de la columna de agua en la estación "C" de la Bahía de Ancón.

de plantas gametofíticas como tetrasporofíticas fue menor de 100 ind. $\times 32 \mathrm{~m}^{-1}$ de línea de orilla, con un incremento entre agosto $y$ setiembre. A inicios de 1997, estas densidades llegaron a valores mucho mayores y mantuvo este nivel de densidades hasta octubre, cuando se produjo un incremento abrupto, que alcanzó - 1815 individuos gametofíticos y 869 tetrasporofíticos en $32 \mathrm{~m}$ de línea de orilla (Fig. 4). Posteriormente, los valores de densidad empezaron a descender llegando hasta sólo 6 plantas gametofíticas y ausencia de tetrasporofíticas en enero de 1998. Esta caída de la población coincidió con la proliferación de las poblaciones de los opistobranquios Aplysia inca y A. nigra. Los valores bajos de densidad se mantuvieron durante la primera mitad de 1998, y en agosto y setiembre se manifestó un evidente incremento.

La proporción de gametofitos varió entre 

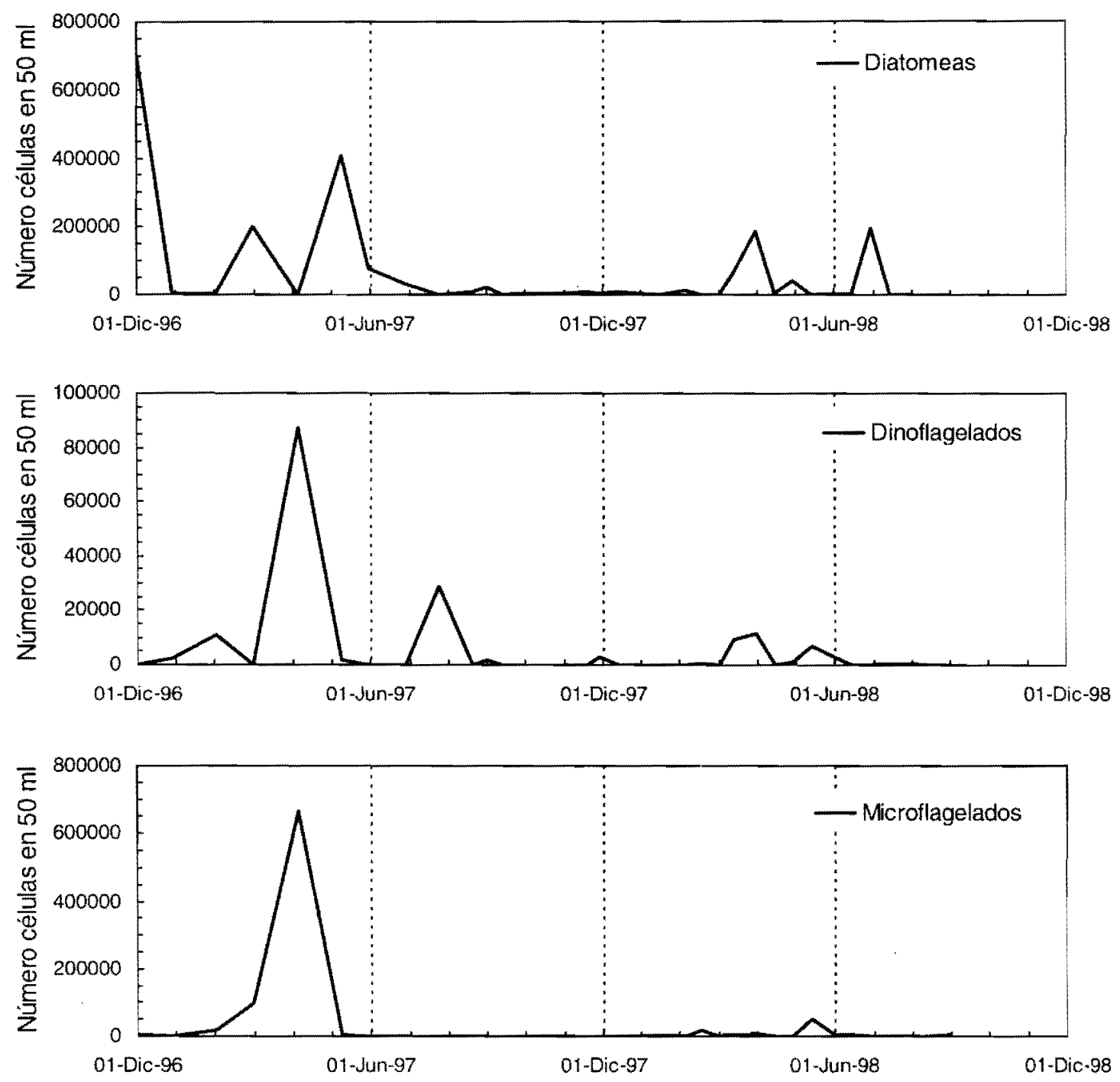

Figura 3. Variaciones de la densidad de diatomeas, dinoflagelados y microflagelados del fitoplancton superficial en la estación "B" de la Bahía de Ancón.

el 55 y $75 \%$ de la población en 1996. Durante 1997 el incremento estacional de la proporción de gametofitos en el segundo semestre se retardó hasta noviembre; y a inicios de 1998 llegó hasta el 100\% (Fig. 4).

\section{Macrobentos de fondo blando}

La fauna bentónica en los fondos blandos de 34 metros de profundidad de la Bahía de
Ancón, al empezar el período de estudio, fue escasa hasta inicios de mayo de 1997. alcanzando un máximo de 5 especies, a fines de mayo fue de 7 y a finales de noviembre llegó hasta 20, que fue el máximo número de especies registradas durante EN 1997-98 en la comunidad (Fig. 5). A partir de dicha fecha disminuyó bruscamente a 9 especies en diciembre; y durante 1998 fluctuó entre 6 y 12 especies. 

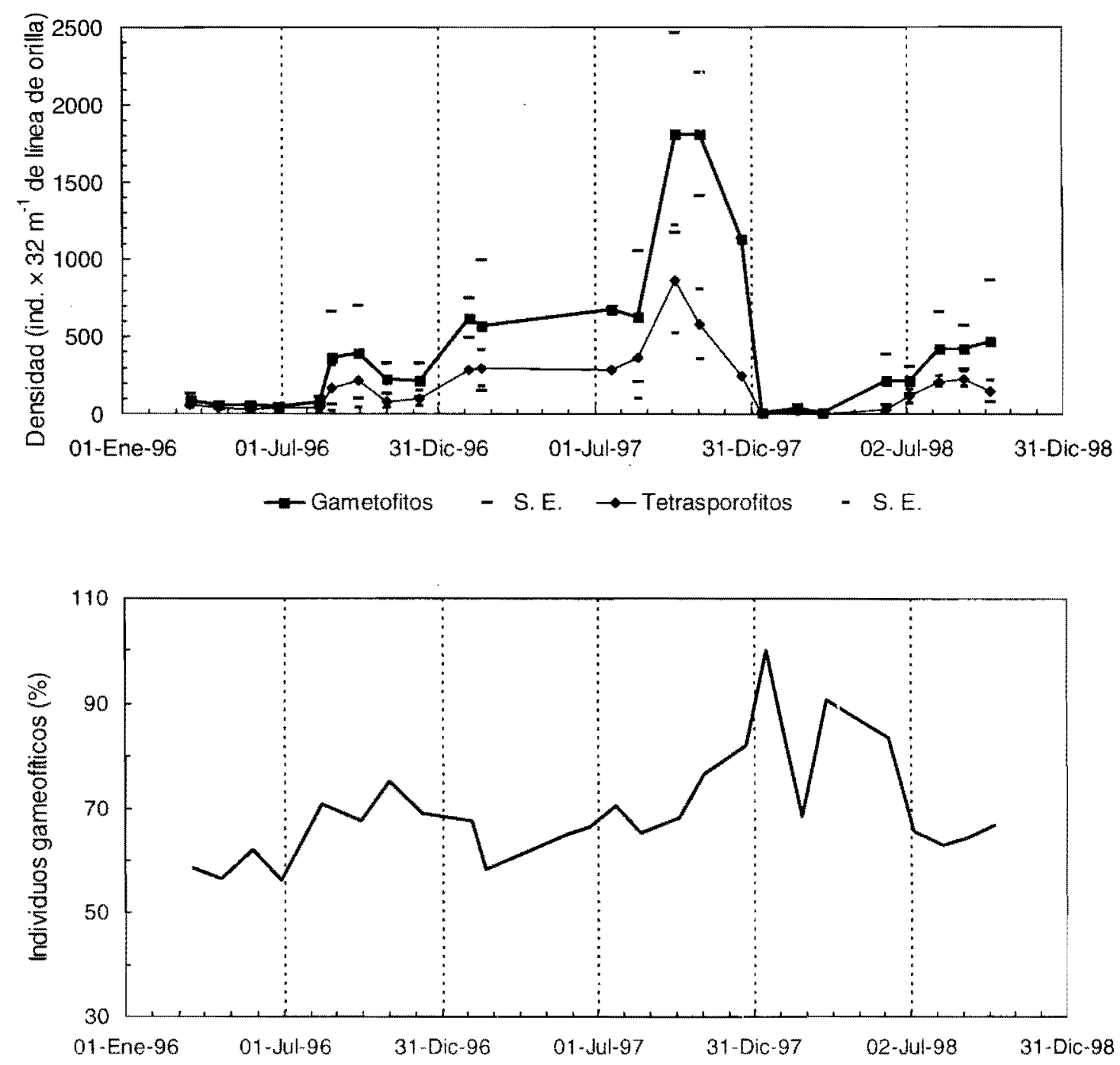

Figura 4. Variaciones de la densidad de individuos gametofíticos y tetrasporofíticos (arriba) y porcentaje de gametofitos (abajo) en la población del alga roja Chondracanthus chamissoi, en la orilla rocosa de la Bahia de Ancón.

La densidad y biomasa de la comunidad macrobentónica mantuvieron valores bajos hasta inicios de mayo de 1997, con un máximo de 5,3 ind. $\times 0,04 \mathrm{~m}^{2}$ y $0,4 \mathrm{mg}$ de peso seco sin ceniza (pssc) $\times 0,04 \mathrm{~m}^{2}$, respectivamente. A partir de fines de mayo la densidad aumentó abruptamente, alcanzando un máximo de 90 ind. $\times 0,04 \mathrm{~m}^{-2}$ en junio de 1997 ; $y$ luego se mantuvo fluctuante con valores mayores de 20 ind. $\times 0,04 \mathrm{~m}^{-2}$, hasta el final del periodo de estudio, excepto en febrero de 1998 (Fig. 5). La biomasa también empezó a crecer desde fines de mayo de 1997, pero recién alcanzó su valor máximo de $0,55 \mathrm{~g} \mathrm{pssc} \times 0,04 \mathrm{~m}^{-2}$ en eñero de 1998. En febrero de 1998 la biomasa descendió a valores muy bajos, pero en marzo empezó a recuperarse y luego mantuvo valores mayores de $0,11 \mathrm{~g} \mathrm{pssc} \times 0,04 \mathrm{~m}^{-2}$, hasta el final del período de estudio (Fig. 5).

Los nemátodos y los poliquetos Paraprionospio pinnata y Parandalia fauveli resultaron las especies dominantes en número y biomasa entre diciembre de 1996 y abril de 1997; y a inicios de mayo la comunidad 

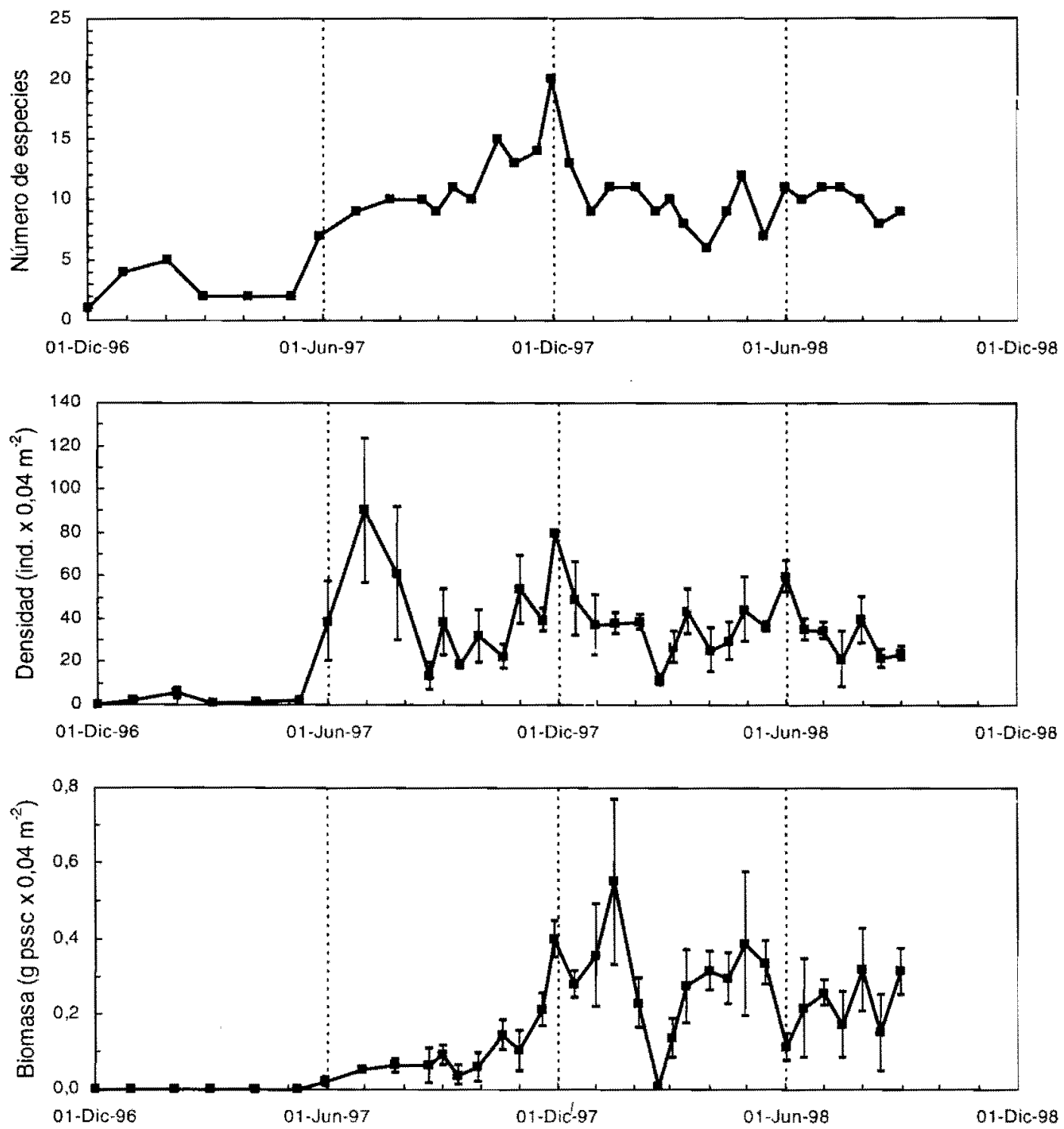

Figura 5. Variación del número de especies, densidad y biomasa (peso seco sin ceniza) en la estación " $\mathrm{C}$ " (34 m de profundidad) de la Bahía de Ancón.

macrobentónica estuvo dominada por el forónido Phoronis sp., tanto en densidad como biomasa. A fines de mayo y en junio de 1997 el poliqueto Capitella capitata fue la especie dominante en número y desde julio de 1997 hasta agosto de 1998 fue dominante el poliqueto Sigambra bassi. La especie dominante en biomasa a fines de mayo de 1997 fue el poliqueto $C$. capitata; y de junio de 1997 hasta agosto de 1998 lo fue un hemicordado, excepto en febrero de 1998, cuando el crustáceo Pinnixa valdiviensis fue la dominante. A fines de noviembre de 1997 se presentó la máxima incursión de especies nuevas y en diciembre el máximo número de especies desaparecidas. Esto último fue debido principalmente a que la mayoría de las especies que colonizaron en noviembre no lograron tener éxito.

Durante el período anterior a EN los organismos bentónicos alimentadores de depósito fueron dominantes tanto en número de especies como en densidad y biomasa. A inicios de mayo de 1997, se produjo una 


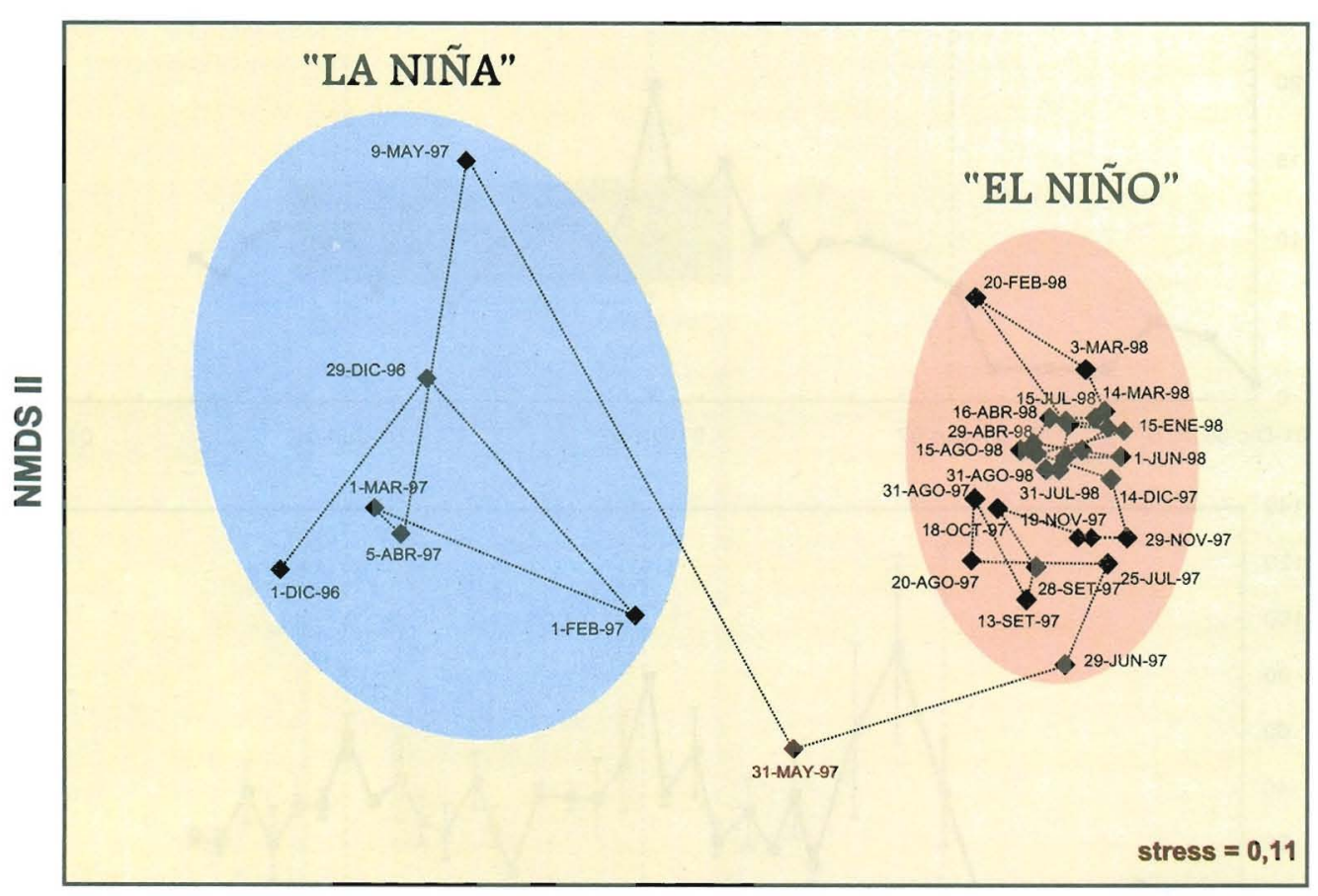

NMDS I

Figura 6. Cambio de un estado "La Niña" a otro "El Niño", mostrado por el análisis de escaleo no métrico multidimensional de la comunidad del macrobentos de la estación " $\mathrm{C}$ " (34 m de profundidad) de la Bahía de Ancón, durante el periodo de diciembre de 1996 hasta agosto de 1998.

alteración en la proporción de dominancia de los grupos tróficos por el incremento en densidad y biomasa de organismos alimentadores de suspensión y a partir de julio de 1997 los carnívoros se hicieron dominantes en número y los alimentadores de suspensión los dominantes en biomasa. A partir de esa fecha estos grupos dominaron tanto en el segundo pico de EN, como en el período posterior a EN. El número de especies durante el primer y segundo pico de EN estuvo dominado, alternadamente, por alimentadores de depósito y alimentadores de suspensión.

\section{Magnitud del Impacto Biológico de EN}

El análisis multivariado de ordenación (NMDS) entre muestras de diferentes fechas discrimina claramente: un estado de la comunidad correspondiente al evento frío " $\mathrm{La}$
Niña" (diciembre de 1996 a inicios de mayo de 1997), caracterizado por una escasa macrofauna y predominio de nemátodos; posteriormente, un breve estado de transición a fines de mayo, dominado por el poliqueto C. capitata; y finalmente el estado EN (junio de 1997 a agosto de 1998), caracterizado por un incremento en densidad, diversidad y biomasa, y el dominio del poliqueto $S$. bassi (Fig. 6).

El índice multivariado de impacto biológico de "El Niño" (IMIBEN) mostró valores negativos hasta abril de 1997 y a inicios de mayo ya había alcanzado valores positivos, indicando claramente la primera respuesta biológica a la presencia de "El Niño 1997-98"; posteriormente, el índice incrementa con el aumento de la temperatura del agua; y al final del calentamiento, desde 


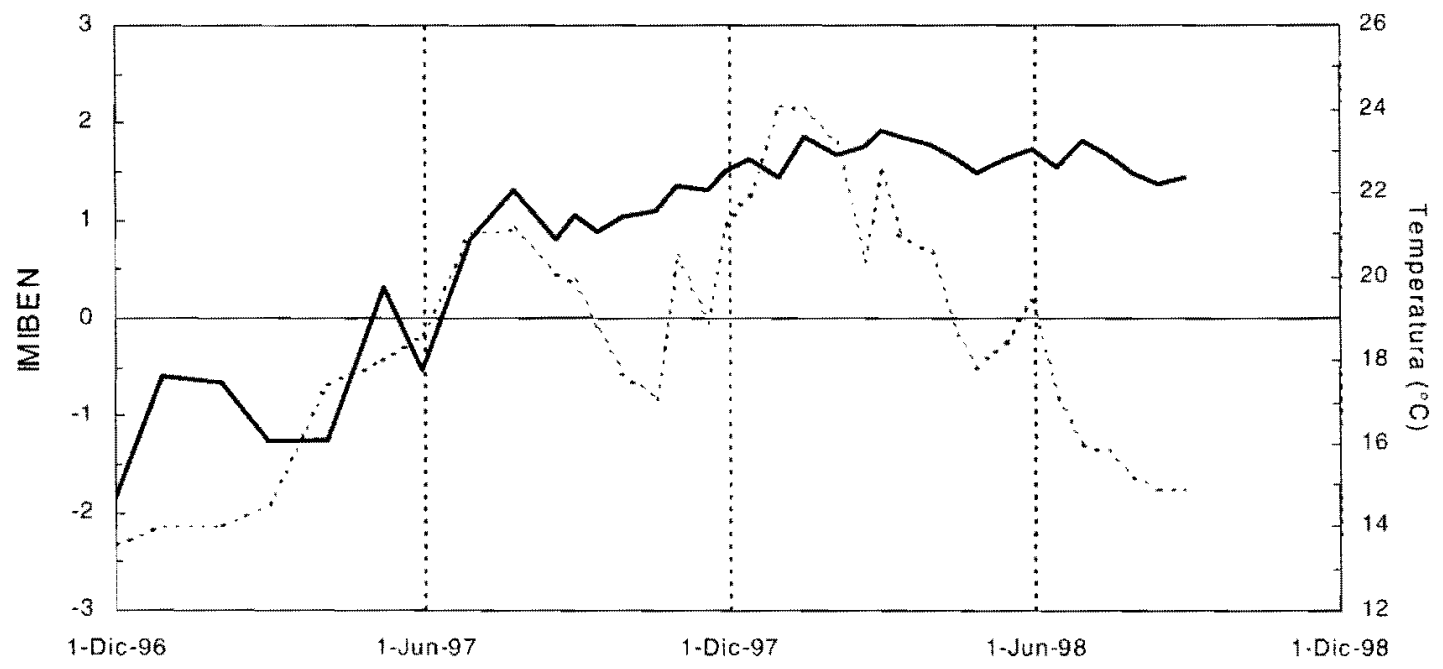

Figura 7. Variaciones del indice multivariado de impacto biológico de "EI Niño" (IMIBEN) y de la temperatura en fondo blando somero de la estación " $\mathrm{C}$ " ( $34 \mathrm{~m}$ de profundidad) en la Bahía de Ancón, durante 1996-98.

junio de 1998, los valores del indice permanecen altos, mostrando el estado post-Niño (Fig. 7), sostenido por las concentraciones todavía altas de oxígeno disuelto en el fondo.
Los análisis de varianza para determinar el efecto de las cuatro etapas empíricamente establecidas, del calentamiento de EN $1997-$ 98 sobre la variación de los parámetros biológicos y oceanográficos demostró un

Tabla 1. Análisis de varianza de una cola y de las pruebas de comparaciones múltiples para los datos oceanográficos y parámetros comunitarios del macrobentos y fitoplancton de las estaciones B y C de la Bahia de Ancón.

\begin{tabular}{|c|c|c|c|}
\hline Parámetro & $F$ & $p$ & $\begin{array}{l}\text { Comparaciones múltiples } \\
\text { Prueba de Scheffe (95\%) }\end{array}$ \\
\hline \multicolumn{4}{|l|}{ Datos oceanográficos } \\
\hline Temperatura en fondo & 49,6 & $<0,001$ & AD N1 N2 \\
\hline Oxígeno disuelto en fondo & $-21,6$ & $<0,001$ & $A D N 2 N 1$ \\
\hline \multicolumn{4}{|l|}{ Fitoplancton } \\
\hline Densidad de fitoplancton total & 20,7 & $<0,001$ & DN2N1A \\
\hline Densidad de diatomeas $(L n)$ & 8,2 & $<0,001$ & D N2AN1 \\
\hline Densidad de dinoflagelados & 7,0 & $<0,001$ & $\bar{D} \overline{N 2 \bar{N} 1 A}$ \\
\hline Densidad de microflagelados & 10,2 & $<0,001$ & N1DN2 A \\
\hline \multicolumn{4}{|l|}{ Macrobentos } \\
\hline Número de especies & 19,4 & $<0,001$ & $A N 1 D N 2$ \\
\hline Densidad & 10,0 & $<0,001$ & $A D N 1 N 2$ \\
\hline Biomasa (peso seco sin ceniza) & 20,9 & $<0,001$ & $A N 1 D N 2$ \\
\hline Indice de diversidad H' & 11,0 & $<0,001$ & $A N 2 N 1 D$ \\
\hline $\begin{array}{l}\text { Indice multivariado de impacto } \\
\text { biológico de EN }\end{array}$ & 239,6 & $<0,001$ & $A \mathrm{N1} D \mathrm{N2}$ \\
\hline
\end{tabular}

A : antes de EN

N1: primer pico de EN
N2: segundo pico de EN

$D$; después de EN 
impacto significativo de EN (Tab. 1). La temperatura y la concentración de oxígeno disuelto cerca al fondo en la estación $\mathrm{C}$ fue significativamente diferente durante EN. La densidad del fitoplancton total, la de dinoflagelados y la de microflagelados en la etapa antes de EN fue significativamente mayor que durante el resto de etapas establecidas para el período de estudio. En el macrobentos de fondo blando los valores del número de especies, densidad y diversidad $\mathbf{H}^{\prime}$ fue significativamente menor antes de EN que en el resto de etapas. La biomasa antes y durante la primera intensificación del calentamiento de EN no muestra diferencia significativa. Igualmente el período después de EN y durante la segunda intensificación de EN no son significativamente diferentes, pero este segundo grupo de períodos sí es significativamente mayor que el primero. El IMIBEN fue significativamente diferente entre todas las etapas excepto entre la segunda intensificación de EN y después de EN.

\section{DISCUSIÓN Y CONCLUSIONES}

El evento "El Niño 1997-98" ha sido considerado uno de los más intensos del presente siglo, similar a los eventos de $1925 \mathrm{y}$ 1982-83. Sin embargo, las características de su desarrollo frente a la costa central del Perú fueron radicalmente diferentes, principalmente al inicio del evento. La mayor parte de los últimos eventos EN mejor estudiados muestran un calentamiento inicial en primavera o inicios del verano en la costa pacífica sudamericana, mientras que EN 1997 98 inició sus manifestaciones a fines del verano e inicios de otoño de 1997 (Jaimes, en este volumen).

En todos los eventos existe como patrón común dos intensificaciones de calentamiento, parcialmente influenciados por la estacionalidad. En el caso particular del calentamiento de EN 1997-98 las anomalías de la primera intensificación de calentamiento se produjeron en otoño de 1997 mostrando, anomalías térmicas positivas excepcionalmente elevadas. Sin embargo, estas excepcionales anomalías representaron valores absolutos de temperatura que no excedieron en mucho los valores alcanzados "normalmente" durante el verano (Tarazona et al., 1985a, 1988a), pudiendo ser, por lo menos en parte, la causa de las respuestas biológicas débiles observadas en la primera mitad de 1997.

En algunos trabajos anteriores acerca del subsistema bentónico en el área de estudio (Rosenberg et al., 1983; Tarazona, 1984; Arntz y Tarazona, 1990; Arntz et al., 1991) se ha demostrado que junto con las anomalías térmicas existen otros parámetros importantes como la concentración de oxígeno disuelto.

Generalmente, se reconoce como indicadores biológicos de EN a especies que muestran como respuesta cambios en su distribución geográfica. En los últimos años se ha demostrado que también muchos parámetros de la estructura comunitaria pueden constituir buenos indicadores (Tarazona et al., 1988b; 1996; CPPS, 1993). Algunos autores han destacado la presencia de especies indicadoras tempranas (Chirinos de Vildoso, 1976; Barber y Chávez, 1983; Arntz, 1986; Rojas de Mendiola, 1985; Arntz y Fahrbach, 1996), que no sólo se manifiestan al mismo tiempo en que se produce el calentamiento de EN, sino que en algunos casos se llega a anticipar hasta seis meses como en el caso del bentos durante EN 1982-83 (Tarazona et al., 1988b; 1996).

Tradicionalmente, se considera la presencia de especies oceánicas en el fitoplancton como uno de los mejores indicadores de EN en las aguas costeras (Rojas de Mendiola et al., 1985). Durante EN 1997-98, el fitoplancton fue un buen indicador temprano en la Bahía de Ancón, con presencia de especies oceánicas indicadoras desde enero de 1997 e incrementos marcados en la densidad de dinoflagelados y microflagelados desde abril. Ambos hal lazgos estuvieron relacionados a los primeros desplazamientos registrados para las masas de 
agua frente a la costa norte y centro, al inicio del evento EN 1997-98 (Jaimes, en este volumen). Considerando que el primer calentamiento de EN se presentó entre julio y agosto de 1997, el macrobentos de fondo blando tuvo también una respuesta positiva temprana, evidenciada por el incremento en la incursión de especies nuevas desde fines de mayo.

Aún no son muy claros los mecanismos y procesos implicados en las respuestas tempranas al impacto de EN (Santander y Flores, 1983; Arntz, 1984; Barber y Chávez, 1986). Sin embargo, las características de los cambios en las comunidades del macrobentos en el área de estudio demuestran indirectamente la presencia de una serie de mecanismos y procesos, como: los cambios en la circulación de las masas de agua con el desplazamiento de aguas ecuatoriales más de $10^{\circ}$ de latitud hacia el sur, evidenciado por el acarreo de formas larvales y adultos de una serie de especies asociadas al plancton; los diversos procesos físiológicos y bioquímicos relacionados al crecimiento y reproducción, asociados al incremento de temperatura $y$ oxígeno observados; las alteraciones en las interacciones biológicas de competencia y depredación, ocasionadas por la proliferación de algunas especies emigrantes y la declinación o proliferación de especies locales (Tarazona et al., 1985b); y la alteración de los procesos de sucesión ecológica, demostrados por la aparición de nuevas especies dominantes en algunas comunidades y la manifestación de nuevos puntos de estabilidad en las comunidades (Sutherland, 1974).

El seguimiento de la población del alga roja C. chamissoi demostró la importancia de las interacciones biológicas durante EN, cuando el impacto positivo en su densidad se convirtió en negativo por la actividad pastoreadora de las poblaciones de los opistobranquios Aplysia nigra y $A$. inca, que proliferaron también a consecuencia de EN (Córdova et al, 1998).

No obstante que las anomalías oceanográficas fueron igualmente intensas durante los eventos EN 1982-83 y 1997-98, en general, el impacto biológico del último fue casi 5 veces menor comparado con los valores obtenidos durante EN 1982-83 tanto en el plancton como en el bentos de las aguas someras de la Bahía de Ancón (Tarazona, 1984; Tarazona et al., 1985b, 1988a, 1996).

Al igual que en eventos pasados, EN 1997 98 mostró también un período post-Niño cuya manifestación se hizo más evidente al término de las anomalías térmicas positivas entre mayo y junio de 1998 (Jaimes, en este volumen). En el presente trabajo, el índice multivariado del impacto biológico de EN determinó cuantitativamente la magnitud del impacto biológico post-Niño. La importancia práctica de dicho índice es discutido en otro trabajo (Tarazona et al., en prep.). Este período estaría condicionado por un conjunto de mecanismos que mantienen por un tiempo a la comunidad en estado Niño una vez terminadas las anomalías térmicas positivas (Arntz et al. 1985; Tarazona et al., 1988a). En el caso del macrobentos de fondo blando de la Bahía de Ancón se ha determinado que esta etapa parece estar influenciada, en parte, por la presencia de pulsos de concentraciones de oxígeno disuelto compatibles con la vida (Tarazona et al., 1991).

Una serie de hallazgos demuestran que los cambios en la circulación de las masas de agua y la profundización de la termoclina producidos por EN estarían determinando nuevos flujos génicos y una mayor probabilidad de hibridización entre poblaciones (Tarazona, 1994; Tarazona y Valle, 1999). Sin embargo, se requiere de mayor investigación para discriminar la magnitud de dichos cambios y establecer la medida en que estos pueden significar impactos biológicos positivos o negativos en el ecosistema marino somero.

En el presente trabajo, en las áreas someras, se ha demostrado señales oceanográficas y biológicas inducidas por EN similares a los registrados en mar abierto (Gutiérrez, 1998); además, también buenos indicadores 
biológicos tempranos, lo cual resulta muy importante en el diseño estratégico de monitoreo en áreas someras, que serían menos costosas y logísticamente más fáciles de desarrollar que en mar abierto.

En general, podemos concluir que: el impacto biológico del evento EN 1997-98 fue menor al de EN 1982-83, no obstante que la intensidad de ambos eventos fue similar; en las áreas someras existen indicadores tan tempranos de EN como en mar abierto; tanto el fitoplancton como el macrobentos de fondo blando presentan especies indicadoras tempranas de EN confiables; la estructura comunitaria del macrobentos también demostró ser un buen indicador de la magnitud del impacto post-Niño; las anomalías oceanográficas han terminado, pero las consecuencias negativas o positivas se mantienen hasta el término del período de estudio; $y$, se ha demostrado que el impacto del evento EN sobre una población o comunidad puede resultar compleja, por el efecto combinado de los disturbios mecánicos - las alteraciones químicas que genera el evento, pudiendo transformar un efecto positivo en negativo y viceversa, durante el mismo evento EN y aún después de éste.

Agradecimientos: A los miembros del grupo DePSEA de la Fac. de Ciencias Biológicas-UNMSM por su colaboración en los trabajos de campo. El estudio ha sido financiado por la Organización de Estados Americanos; el Alfred Wegener Institut für Polar und Meeresforschung de Bremerhaven, Alemania; el Consejo Nacional de Ciencia y Tecnología y por el Fondo de Desarrollo Universitario (UNMSM).El presente artículo es la contribución $N^{\circ} 1776$ del Instituto Alfred Wegener de Investigaciones Polares y Marinas.

\section{LITERATURA CITADA}

Arntz, W. 1984. El Niño and Perú: Positive aspects. Oceanus 27 (2): 36-39. 1986. The two faces of El Niño 1982-83.
Meeresforsch 31: 1-46.

Arntz. W. y E. Fahrbach. 1996. El Niño: Experimeno climático de la Naturaleza. Fondo de Cultura Económica, Mexico, 312 pp

Arntz, W. \& J. Tarazona. 1990. Effects of EI Niño $1982-$ 83 on benthos, fish and fisheries off the South American Pacific coast. In: P.W. Glynn (ed.). Global ecological consequences of the 1982 . 83 El Niño-Southern Oscillation. Elsevier Oceanography Series (52): 323-360.

Arntz, W.; A. Landa y J. Tarazona (eds.). 1985, "El Niño", su impacto en la fatma marina. Bol. Inst. Mar Perú-Callao Vol. Extraor., Callao. Perú, 22 pp.

Arntz, W.; J. Tarazona: V.A. Gallardo: L.A. Flores \& H. Salzwedel. 1991. Benthos communities in oxygen deficient shelf and upper slope areas of the Peruvian and Chilean Pacific coast, and changes caused by El Niño. M: R. V. Tyson \& T. H. Pearson (eds.). Modern and Ancient Continental Shelf Anoxia. Geological Society Special Publication 58: 131-154.

Barber, R.T. \& E.P. Chávez. 1983. Biological consequences of El Niño. Science 222: 1203 1210 .

-1986. Ocean variability in relation to living resources during the $1982-83 \mathrm{El}$ Niño. Natate 319: 279-285.

Barber, R.T. \& J.E. Kogelschatz. 1990. Nutrients and productivity during the 1982/83 El Niño. In: P.W. Glynn (ed.). Global ecological consequences of the 1982.83 El NiñoSouthern Oscillation. Elsevier Oceanography Series (52): $21-53$.

Carrasco, S. \& O. Lozano. 1989. Seasonal and longm term vatiations of zooplankton volumes in the Peruvian Sca, 1964-1987. In: D. Pauly: P. Muck; J. Mendo \& Tsukuyama (eds.). The Peruvian upwelling ecosystem: dynamics and interactions. ICLARM Conference Procectings (18): 82-85.

Córdova, C.: T. Peña: O. Riofrio; Y, Reyes: C. Magallanes y L. Romero. 1998. Variación de la cobertura do especies en los parches intermareales de Chondractuhtus chamissot, durante "El Niño" 1997-98. Seminario-Taller: "El Niño" en América Latina. sus impactos biológicos y sociales: Bases para un monitoreo regional, Lima, Perú. Libro de Resimenes, p. 12.

CPPS (Comisión Pcrmanente del Pacifico Sur). 1993. Curso Taller Intentacional sobre indicadores biológicos del fenómeno El Niño en el Pacifico Sudeste. Cali. Colombia, marzo 1993. Santiago, Chile, 38 pp.

Chirinos de Vildoso. A. 1976. Aspectos del fenómeno El Niño 1972-73. Parte 1; Distribución de la fauna. FAO Informes de Pesca 185: 62-79. 
Enfield, D. \& L. Cid. 1991. Low-frecuency changes in El Niño-Souther Oscillation. J. Climate 4: $1137-1146$

Espino, M.; C. Benites y M. Maldonado. 1985. Situación de la población de merluza (Merluccius gayi peruanus) durante "El Niño". En: W. Arntz; A. Landa y J. Tarazona (eds.) "El Niño", su impacto en la fauna marina. Bol. Inst. Mar Peru-Callao Vol. Extraor: 159-162.

Fasham, M.J.R. 1977. A comparison of non-metric multidimensional scaling, principal components and reciprocal averaging for the ordination of simulated coeclines and coeplanes. Ecology 58:551-561.

Garbary, D. \& R.E. DeWreede. 1988. Life history phases in natural populations of Gigartinaceae (Rhodophyta): quantification using resorcinol. In: C.S. Lobban; D.J. Chapman \& B.P. Kremer (eds.). Experimental Phycology. A Laboratory Manual. Cambridge University Press, pp. 174-178

Gutiérrez, M. 1998. Evidencias del efecto de "El Niño 1997-98" sobre la distribución y abundancia de la anchoveta (Engraulis ringens). Seminario-Taller: "El Niño" en América Latina, sus impactos biológicos y sociales: Bases para un monitorco regional, Lima. Perú. Libro de Resimenes, p. 22.

Majluf, P. \& F. Trillmich. 1981. Distribution and abundance of sea lions (Otaria byronia) and fur seals (Arctocephalus austratis) in Peru. $Z$. f. Säugetierkunde 46 (6): 384-393.

Prentice, 1.C. 1977. Non-metric ordination methods in ecology, J. Ecol. 65: 85-94.

Rither, J.H. 1969. Photosynthesis and fish production in the sea. Science 166: 72-76.

Rojas de Mendiola, B.; O. Gómez y N. Ochoa. 1985. Efectos del tenómeno "El Niño" sobre el fitoplancton. En: W. Arntz; A. Landa y J. Tarazona (eds.). "El Niño", su impacto en la fauna marina. Bol. Insi. Mar Peni-Callao Vol. Extraor: $33-40$.

Rosenberg, R, W. Arntz; E. Chumän de Flores; L.A. Flores; $G$. Carbajal; 1. Finger \& J. Tarazona. 1983. Benthos biomass and oxygen deficiency in the upwelling system off Peru. $J$ Mar. Res. 41: 263-279

Santander, H. y R. Flores. 1983. Los desoves y distribución larval de cuatro especies pelágicas $y$ sus relaciones con las variaciones del ambiente marino frente al Perú. FAO Fish. Rep. 291 (3): 835-867.

Santander. H. \& I. Tsukayama. 1984. The anchoveta and sardine and some events associated to their recruitment. IOC Worksh. Rep. 33: 11-14.

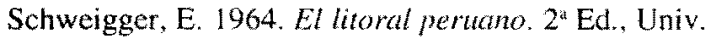
Nac. Foderico Villareal, Lima, $414 \mathrm{pp}$.
Shannon, C. \& W. Weaver. 1949. The mathematical theory of communication. Univ. of Illinois Press, Urbana, 117 pp.

Sneath, P.H.A. \& R.R. Sokal. 1973. Ninerical taxonomy: W. H. Freeman, San Francisco, 571 pp.

Sutherland, J.P. 1974. Multiple stable points in natural communities. An. Nat. 108: 859-873.

Tarazona, J. 1984. Modificaciones de la infauna bentónica de una bahía con deficiencia de oxígeno durante "El Niño" 1982-83. Rev. Com. Perm. Pacifico Sur 15: 223-238.

Tarazona, J. 1994. Biodiversidad en el Mar Peruano. En: Actas. Foro Anual: Biodiversidad y Desarrollo Sustentable. Academia Nacional de Ciencia y Tecnología. Tomo 2 (3): 9-12.

Tarazona, J. y Valle, S. 1999. La diversidad biológica en el mar peruano. En: G. Halffter (ed.). La diversidad Biológica de Iberoamérica II. CYTED-D, Instituto de Ecología, Xalapa. México, pp. 103-115.

Tarazona, J.; H. Salzwedel \& W. Arntz. 1988a. Possitive effects of "El Niño" on macrozoobenthos inhabiting hypoxic areas of Peruvian upwelling system. Oecologia 76: 184-190.

- 1988b. Oscillations of macrobenthos in shallow waters of the Peruvian central coast induced by $\mathrm{E}$ Niño 1982-83. J. Mar. Res. 46: $593-611$.

Tarazona. J.: W. Arntz \& E. Canahuire. 1996. Impact of two "El Niño" events of different intensity on the hypoxic soft bottom macrobenthos off the central Peruvian coast. Marine Ecology $17(1-3): 425-446$

Tarazona, J.; W. Arntz; E. Canahuire: Z. Ayala y A. Robles. 1985a. Modificaciones producidas durante "El Niño" en la infauna bentónica de áreas someras del ecosistema de afloramiento peruano. En: W. Arntz; A. Landa y J. Tarazona (eds.). "El Niño", su impacto en la fauna marina. Bol. Inst. Mar Perí-Callao Vol. Extraor.: 55-63

Tarazona, J; C. Paredes; L. Romero; V. Blascovich: S. Guzmán y S. Sánchez. 1985b. Características de la vida planctónica y colonización de los organismos bentónicos epilíticos durante el fenómeno "El Nin̆o", En: W. Amtz: A. Landa y J. Tarazona (eds.). "El Niño", su impacto en la fauna marina. Bol. Inst. Mar Perí-Calloo Vol. Extraor: $41-49$

Tarazona. J; E. Canahuire; $H$. Salzwedel; T. Jeri; W. Arntz \& L. Cid. 1991. Macrozoobenthos in two shallows areas of Peruvian upwelling ecosystem. In: M. Elliott \& J.P. Ducrotoy (eds.) Estuaries and Coasts: Spatial and Temporal Intercomparisons. ECSA 19 Symposium: 251-258. 